\title{
National Survey of Fitness Trends in Greece for 2021
}

\author{
Alexios Batrakoulis ${ }^{1, *}$, Athanasios Chatzinikolaou ${ }^{2}$, Athanasios Z. Jamurtas ${ }^{1}$, Ioannis G. Fatouros ${ }^{1}$ \\ ${ }^{1}$ School of Physical Education and Sport Science, University of Thessaly, Trikala, Greece \\ ${ }^{2}$ School of Physical Education and Sport Science, Democritus University of Thrace, Komotini, Greece
}

Received August 30, 2020; Revised October 14, 2020; Accepted November 11, 2020

\begin{abstract}
Cite This Paper in the following Citation Styles
(a): [1] Alexios Batrakoulis, Athanasios Chatzinikolaou, Athanasios Z. Jamurtas, Ioannis G. Fatouros, "National Survey of Fitness Trends in Greece for 2021," International Journal of Human Movement and Sports Sciences, Vol. 8, No. 6, pp. 308 - 320, 2020. DOI: 10.13189/saj.2020.080602.
\end{abstract}

(b): Alexios Batrakoulis, Athanasios Chatzinikolaou, Athanasios Z. Jamurtas, Ioannis G. Fatouros (2020). National Survey of Fitness Trends in Greece for 2021. International Journal of Human Movement and Sports Sciences, 8(6), 308 320. DOI: $10.13189 /$ saj.2020.080602.

Copyright $\odot 2020$ by authors, all rights reserved. Authors agree that this article remains permanently open access under the terms of the Creative Commons Attribution License 4.0 International License

\begin{abstract}
The objective of this study was to identify the most important health/fitness trends while examining the impact of various demographics of respondents on the top trends for the first time in Greece. An online survey was sent to 3,487 professionals who worked in the health/fitness sector using the methodology of similar regional and worldwide studies extensively conducted by the American College of Sports Medicine (ACSM). A total of 542 responses were collected with a response rate of $15.5 \%$. The ten most important trends were personal training, exercise is medicine, high-intensity interval training, small group training, and functional fitness, body weight training, exercise for weight loss, circuit training, strength training with free weights, and fitness programs for older adults. Health-related trends appear to be popular while technology- and mind/body-related trends seem not to be very attractive. Traditional exercise modes are still attractive and relevant, but specialized group training approaches are currently experiencing low popularity. Results also showed meaningful sex differences for $68 \%$ of the examined trends where female respondents reported higher scores. The main findings of this study are in line with those recently reported in the ACSM's European survey even though similar research design and data analysis are limited internationally.
\end{abstract}

Keywords Greek Fitness Survey, Fitness Sector, Trends, Top Programs, ACSM Survey

\section{Introduction}

The fitness sector has grown tremendously in the last decades at both European [1] and global level [2] demonstrating significant increased number of members and facilities. As such, it has been considered an exciting and innovative sector showing great potential for continuous growth while offering promising professional opportunities and business development concepts [3]. However, this sector is addressed to consumers classified as sedentary and overweight because one in two adults does not meet the recommended physical activity guidelines [4] while two in three of them do not have a healthy weight [5] worldwide.

In Greece, it has been estimated that there are approximately 1,470 commercial fitness clubs, 0.735 million members, annual revenues of EUR $€ 0.37$ billion, and a penetration rate of $6.68 \%[6,7]$. Greece has been reported as a country demonstrating one of the finest health statuses among other European countries [8]. Despite the fact that the prevalence of chronic conditions is systematically growing nationwide resulting into a slower increase in life expectancy compared to the mean of the European Union (EU) countries [9]. Specifically, it has been recently estimated that $60 \%, 27 \%, 9 \%, 26 \%$ of the Greek adult population has an unhealthy weight, obesity, raised fasting blood glucose and raised resting blood pressure, respectively [8]. Additionally, physical inactivity is increasing at an alarming rate and thus Greece demonstrates one of the highest rates of inactivity in the EU [10]. It is notable that $68 \%$ of adults do not exercise or 
participate in any physical or sport activity on a weekly basis. [10]. Moreover, only $20 \%$ of the adult population meets the recommended physical activity levels [11] and only $5 \%$ of them are fitness club members [6,7]. Interestingly, $22 \%$ of adults who engage in regular physical activity prefer fitness centers for their exercise experience, which is a higher rate than that observed among Europeans $(15 \%)$ [10]. It also notable that fitness is now the number one participation sport in Europe [10] showing that this sector is systematically growing in the region.

On the other side, the vital role of regular exercise in preventing, managing and treating lifestyle-related chronic diseases that immensely affect public health nowadays has been extensively documented [12]. Furthermore, the changing role of exercise professionals in the fitness and clinical settings has been recently reported as a critical link between the physical activity guide pyramid and community aiming to promote the value of active lifestyle in health, well-being and physical performance among various populations of all ages [13]. The American College of Sports Medicine (ACSM) has established a worldwide annual survey on fitness trends since 2006 aiming to identify the most popular, valuable and safe physical activity and exercise modes among all involved stakeholders [14]. Such an investigation may support exercisers engagement through a positive fitness experience while enhancing professional development, creating novel business concepts, and enhancing service quality in the health and fitness sector. [14].

\section{Objectives}

No study has yet surveyed in-depth the state of fitness trends in Greece. On the other hand, the ACSM's survey has been including data from different regions providing insights into regional and international comparisons [15]. Moreover, a recent European survey investigating fitness trends by collecting data from 40 different countries is not capable to produce secure remarks for Greece because of its diverse sample sizes and the limited recruitment of Greek participants [16]. Thus, the present study aims a) to identify important trends in the health and fitness sector in Greece for 2021, b) to examine the effects of various demographic characteristics on health and fitness trends at national level, and c) to compare the results obtained with those identified by other recent international surveys for fitness trends. The results of this survey may contribute to decisions made in the fitness, health, and wellness markets supporting the fight against the obesity and inactivity epidemics nationwide.

\section{Materials and Methods}

\subsection{Study Design}

The present study used the ACSM's methodology applying exactly the same criteria with those that have been widely used since 2006 in the worldwide [14], North American [15], European [16], Asian [17], and South American [18] surveys of fitness trends. The survey was designed to identify the trends (not fads) that are considered attractive because they may have a positive impact on the local fitness industry while demonstrating high popularity among fitness industry stakeholders who are based in Greece working with several types of populations. Thus, a distinction between a "fad" and a "trend" according to dictionary was included in the introduction of the survey to help respondents understand the difference between these two key words. The potential impact of products, services, or equipment on the Greek fitness market was not evaluated by this national survey. In brief, a team of experts in commercial, community, corporate, and clinical health/fitness sectors as well as academia was recruited by the ACSM to help identify a list of previously identified trends as well as some new emerging trends [14]. As such, an electronic-based survey was developed including 41 applicable trends in the health and fitness sector that were identified from various sources and from the personal experience of some experts. Each potential trend was accompanied by a brief explanation that provided respondents with a basic description in English as previously reported [16]. The questionnaire also included 10 demographic questions and was designed to be completed in 15 minutes or less and all information was provided in English without any change from the original edition developed by ACSM. The research was conducted electronically from April 7 to June 2, 2020 (8 weeks) and no incentives were offered to help increase participation in the survey. The submitted responses on the potential trends were assessed using a 10-point Likert scale ranging from 1 (least likely to be a trend) to 10 (most likely to be a trend) as previously described [14].

\subsection{Participants}

Databases of contacts of local universities i.e., Schools of Physical Education and Sport Sciences across the country as well as the Panhellenic Union of Physical Education Teachers, Panhellenic Gyms Association and the European Register of Exercise Professionals (only Greek registered members) were mainly used to recruit respondents for this survey. The online survey was sent electronically to 3,487 individual contacts in total. All contacts were health and fitness industry stakeholders who lived and worked in Greece such as exercise professionals of any employment status, university students (undergraduate and postgraduate level), faculty and teaching staff members, gym owners/operators, managers and program directors. Likewise, the survey was posted on web sites and social media accounts of all involved parties nationwide. 


\subsection{Statistical Analyses}

The Shapiro-Wilk test was used to determine data normality. Descriptive statistics are presented as a proportion (\%) for demographics and as a mean score (0-10) \pm standard deviation (SD) for the trends ranking. Mauchly's test of sphericity was examined to test for equality of variance, Levene's test was conducted for the assumption of homogeneity of variance and an independent $t$ test was used to determine differences between female and male respondents for each trend. Differences between qualified and non-qualified respondents in personal training, group fitness, and exercise for health were also determined by a $t$ test. The one-way analysis of variance (ANOVA) test was used to determine differences in the various trends by all demographic characteristics apart from gender. When a significant interaction was detected, multiple comparison analysis was conducted using a Bonferroni post-hoc test. Statistical significance was set at $p<0.05$ and confidence intervals (CI) were also calculated. Data were analyzed using the SPSS 24.0 software (IBM Corp., Armonk, NY, USA) and are presented as mean \pm standard deviation (SD).

\section{Results}

The online survey collected 542 responses, which represents a return rate of $15.5 \%$. Demographic characteristics are shown in Table 1. All candidate trends were ranked from highest (most popular trend) to lowest (least popular trend) and are illustrated in Table 2. Statistically significant differences between female and male respondents for all trends are shown in Table 3.

In brief, there was a significant difference in the mean score between female and male for 28 out of 41 fitness trends (68.3\%). Specifically, female respondents reported a higher score $(+12 \%, p=0.001-0.035)$ than men for all trends apart from low-cost and budget gyms (-10\%, $p=0.012$ ) (Table 3). Due to the large amount of data, differences only among the top 20 trends and demographics (age group, education, qualifications, primary profession, experience, work status, and career choice) as well as respondents' qualifications are presented in Tables 4 and 5, respectively. Results for trends ranked from the 21 st to 41 st place are however available from the authors upon reasonable request.

Young age group (18-21 years) demonstrated a markedly lower mean score $(-15 \%, p=0.003-0.024)$ for circuit training and a higher score $(+7 \%, p=0.002-0.047)$ for strength training with free weights than middle-aged participants (35-54 years). In addition, younger respondents (18-34 vs. 55-64 years) presented significantly higher mean ratings $(+35 \%, p=0.006)$ for outdoor activities. Bachelor's degree holders presented a significant lower rating $(-12 \%, p=0.016)$ than doctorate degree holders for children and exercise. Strength training with free weights showed meaningful differences between respondents with lower experience levels (0-5 yrs vs. 10-20 yrs, +9\%, $p=0.020$ ), whereas fitness programs for older adults demonstrated the opposite $(0-5$ yrs vs. $20+$ yrs, $-11 \%$, $p=0.011)$ compared to highly experienced survey participants. Full-time occupations presented markedly higher mean scores for exercise is medicine $(+6 \%$, $p=0.003)$, small group training $(+6 \%, p=0.010)$, and circuit training $(+9 \%, p<0.001)$, whereas part-time work status exhibited significant higher mean ratings for strength training with free weights $(+8 \%, p=0.002)$ and outdoor activities $(+10 \%, p=0.003)$. Hourly workers showed an impactful difference only in strength training with free weights compared to full-time work status $(+12 \%$, $p=0.027$ ). First job demonstrated noticeable difference $(+7 \%, p=0.025)$ in the mean score of exercise for weight loss compared to second job, whereas second job showed substantial differences in the mean ratings of group training $(+7 \%, p=0.037)$, wearable technology $(+10 \%, p=0.004)$, and outdoor activities $(+13 \%, p<0.001)$ in comparison with the first job. Third job displayed marked differences in the mean scores for children and exercise $(+12 \%, p=0.032)$ and outdoor activities $(+18 \%, p=0.001)$ compared to first job (Table 4).

Participants holding the personal training qualification stated meaningful higher scores $(+9 \%, p=0.001-0.032)$ for five out of 20 top tends (e.g., personal training, high-intensity interval training, small group training, circuit training, and boutique fitness studios). On the other hand, unqualified participants in personal training reported a significant higher mean score $(+7 \%, p=0.001-0.019)$ for five trends included in the top 20 list (e.g., functional fitness, fitness programs for older adults, wearable technology, outdoor activities, and post rehabilitation classes) (Table 5). Qualified respondents in exercise for health, group fitness, youth fitness, active aging fitness, pre/postnatal fitness, yoga training, and aquatic fitness presented a significant higher mean score $(+9 \%$, $p=0.001-0.033$ ) for six, four, three, two, six, two and two out of 20 top trends, respectively, compared to those with no qualifications in these particular fields (Table 5). 
Table 1. Demographics of the Greek survey respondents

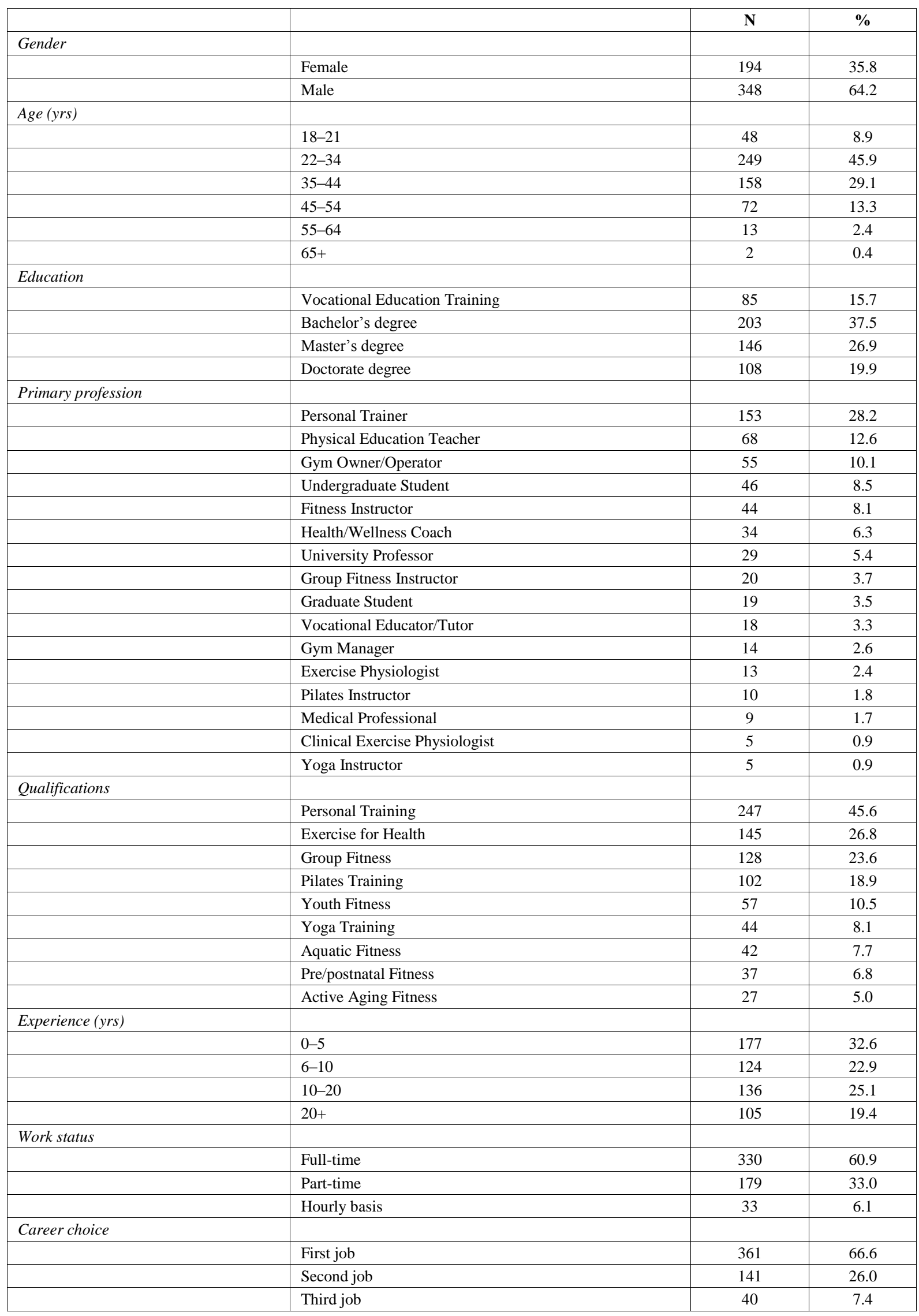


Table 1 Continued

\begin{tabular}{|l|l|c|c|}
\hline & & & \\
\hline & \multicolumn{1}{|c|}{ Annual Salary $(\epsilon)$} & 394 & 72.7 \\
\hline & $20,000-29,999$ & 66 & 12.2 \\
\hline & $30,000-39,999$ & 32 & 5.9 \\
\hline & $40,000-49,999$ & 17 & 3.1 \\
\hline & $50,000-59,999$ & 6 & 1.1 \\
\hline & $60,000-69,999$ & 9 & 1.7 \\
\hline & $70,000-79,999$ & 4 & 0.7 \\
\hline & $80,000-89,999$ & 1 & 0.2 \\
\hline & $90,000-99,999$ & 1 & 0.2 \\
\hline & $>100,000$ & 4 & 0.7 \\
\hline & Not willing to share & 8 & 1.5 \\
\hline
\end{tabular}

Table 2. Comprehensive ranking of fitness trends in Greece for 2021

\begin{tabular}{|c|c|c|}
\hline$\#$ & Trend & Score \pm SD \\
\hline 1 & Personal Training & $8.810 \pm 1.58$ \\
\hline 2 & Exercise is Medicine & $8.804 \pm 1.77$ \\
\hline 3 & High-Intensity Interval Training & $8.328 \pm 1.81$ \\
\hline 4 & Small Group Training & $8.280 \pm 1.84$ \\
\hline 5 & Functional Fitness Training & $8.177 \pm 1.86$ \\
\hline 6 & Body Weight Training & $8.098 \pm 1.84$ \\
\hline 7 & Exercise for Weight Loss & $7.998 \pm 2.04$ \\
\hline 8 & Circuit Training & $7.887 \pm 1.96$ \\
\hline 9 & Strength Training (Free Weights) & $7.620 \pm 2.01$ \\
\hline 10 & Fitness Programs for Older Adults & $7.581 \pm 2.31$ \\
\hline 11 & Wearable Technology & $7.579 \pm 2.42$ \\
\hline 12 & Group Training & $7.574 \pm 2.16$ \\
\hline 13 & Health/Wellness Coaching & $7.563 \pm 2.22$ \\
\hline 14 & Children and Exercise & $7.445 \pm 2.25$ \\
\hline 15 & Employing Certified Fitness Professionals & $7.393 \pm 2.62$ \\
\hline 16 & Outdoor Activities & $7.358 \pm 2.58$ \\
\hline 17 & Post Rehabilitation Classes & $7.341 \pm 2.54$ \\
\hline 18 & Clinical Integration/Medical Fitness & $7.314 \pm 2.31$ \\
\hline 19 & Boutique Fitness Studios & $7.284 \pm 2.36$ \\
\hline 20 & Licensure for Fitness Professionals & $7.247 \pm 2.74$ \\
\hline 21 & Core Training & $7.175 \pm 2.14$ \\
\hline 22 & Pilates & $7.070 \pm 2.34$ \\
\hline 23 & Walking/Running/Jogging/Cycling Clubs & $7.050 \pm 2.43$ \\
\hline 24 & Pre/Post-Natal Fitness & $7.042 \pm 2.54$ \\
\hline 25 & Lifestyle Medicine & $6.838 \pm 2.60$ \\
\hline 26 & Long-term Youth Development & $6.601 \pm 2.78$ \\
\hline 27 & Outcome Measurements & $6.537 \pm 2.56$ \\
\hline 28 & Mobility/Myofascial Devices & $6.518 \pm 2.58$ \\
\hline 29 & Yoga & $6.362 \pm 2.37$ \\
\hline 30 & Online Training & $6.325 \pm 2.77$ \\
\hline 31 & Low-cost and Budget Gyms & $6.129 \pm 2.85$ \\
\hline 32 & Worksite Health Promotion Programs & $6.081 \pm 2.78$ \\
\hline 33 & Boot Camp-Style & $5.948 \pm 2.67$ \\
\hline 34 & Mobile Exercise Apps & $5.834 \pm 2.61$ \\
\hline 35 & Aquatic Exercise & $5.817 \pm 2.74$ \\
\hline 36 & Mind-Body Movement & $5.777 \pm 2.69$ \\
\hline 37 & Worker Incentive Programs & $5.771 \pm 2.76$ \\
\hline 38 & Boxing, Kickboxing \& Mixed Martial Arts & $5.766 \pm 2.63$ \\
\hline 39 & Dance-Based Work-Outs & $5.504 \pm 2.64$ \\
\hline 40 & Virtual Training & $5.218 \pm 2.72$ \\
\hline 41 & Electrical Muscle Stimulation Training & $4.448 \pm 2.37$ \\
\hline
\end{tabular}

$\mathrm{SD}$, standard deviation. 
Table 3. Significant differences among fitness trends and respondents' gender

\begin{tabular}{|c|c|c|c|c|c|c|c|}
\hline \multirow{3}{*}{$\begin{array}{l}\text { Trend } \\
\text { Exercise is Medicine }\end{array}$} & \multirow{3}{*}{$\frac{t}{2.972}$} & \multirow{3}{*}{$\begin{array}{c}\text { df } \\
452\end{array}$} & \multirow{3}{*}{$\begin{array}{c}\boldsymbol{p} \text { value } \\
0.003\end{array}$} & Female & Male & & \\
\hline & & & & \multicolumn{2}{|c|}{ Mean Score \pm SD } & \multicolumn{2}{|c|}{$95 \% \mathrm{CI}$} \\
\hline & & & & $9.09 \pm 1.59$ & $8.64 \pm 1.86$ & 0.152 & 0.746 \\
\hline Functional Fitness Training & 3.294 & 540 & 0.001 & $8.53 \pm 1.73$ & $7.98 \pm 1.90$ & 0.219 & 0.867 \\
\hline Body Weight Training & 2.599 & 540 & 0.010 & $8.37 \pm 1.67$ & $7.95 \pm 1.91$ & 0.104 & 0.748 \\
\hline Circuit Training & 2.424 & 540 & 0.016 & $8.16 \pm 1.78$ & $7.74 \pm 2.04$ & 0.080 & 0.768 \\
\hline Fitness Programs for Older Adults & 6.108 & 503 & 0.001 & $8.30 \pm 1.79$ & $7.18 \pm 2.46$ & 0.764 & 1.488 \\
\hline Health/Wellness Coaching & 4.334 & 540 & $<0.001$ & $8.11 \pm 2.13$ & $7.26 \pm 2.22$ & 0.465 & 1.235 \\
\hline Children and Exercise & 5.428 & 540 & $<0.001$ & $8.13 \pm 2.06$ & $7.06 \pm 2.26$ & 0.680 & 1.451 \\
\hline Outdoor Activities & 4.082 & 436 & $<0.001$ & $7.94 \pm 2.37$ & $7.03 \pm 2.64$ & 0.469 & 1.339 \\
\hline Post Rehabilitation Classes & 6.424 & 469 & $<0.001$ & $8.20 \pm 2.14$ & $6.86 \pm 2.63$ & 0.929 & 1.749 \\
\hline Clinical Integration/Medical Fitness & 3.779 & 540 & $<0.001$ & $7.81 \pm 2.16$ & $7.04 \pm 2.34$ & 0.371 & 1.173 \\
\hline Boutique Fitness Studios & 2.202 & 540 & 0.028 & $7.58 \pm 2.37$ & $7.12 \pm 2.35$ & 0.050 & 0.879 \\
\hline Core Training & 5.181 & 540 & $<0.001$ & $7.80 \pm 2.14$ & $6.83 \pm 2.07$ & 0.603 & 1.340 \\
\hline Pilates & 7.308 & 540 & $<0.001$ & $8.01 \pm 2.19$ & $6.55 \pm 2.26$ & 1.071 & 1.858 \\
\hline $\begin{array}{l}\text { Walking/Running/Jogging/Cycling } \\
\text { Clubs }\end{array}$ & 3.440 & 540 & 0.001 & $7.53 \pm 2.22$ & $6.78 \pm 2.50$ & 0.318 & 1.165 \\
\hline Pre/Post-Natal Fitness & 5.802 & 425 & $<0.001$ & $7.85 \pm 2.35$ & $6.59 \pm 2.54$ & 0.832 & 1.685 \\
\hline Lifestyle Medicine & 4.580 & 540 & $<0.001$ & $7.51 \pm 2.36$ & $6.46 \pm 2.65$ & 0.598 & 1.497 \\
\hline Outcome Measurements & 4.564 & 540 & $<0.001$ & $7.20 \pm 2.51$ & $6.17 \pm 2.51$ & 0.585 & 1.468 \\
\hline Mobility/Myofascial Devices & 4.116 & 434 & $<0.001$ & $7.10 \pm 2.38$ & $6.19 \pm 2.63$ & 0.476 & 1.345 \\
\hline Yoga & 6.203 & 540 & $<0.001$ & $7.18 \pm 2.27$ & $5.91 \pm 2.31$ & 0.871 & 1.679 \\
\hline Online Training & 2.110 & 540 & 0.035 & $6.66 \pm 2.70$ & $6.14 \pm 2.80$ & 0.036 & 1.008 \\
\hline Low-cost and Budget Gyms & -2.531 & 540 & 0.012 & $5.72 \pm 2.81$ & $6.36 \pm 2.85$ & -1.142 & -0.144 \\
\hline $\begin{array}{l}\text { Worksite Health Promotion } \\
\text { Programs }\end{array}$ & 5.604 & 540 & $<0.001$ & $6.95 \pm 2.65$ & $5.59 \pm 2.74$ & 0.882 & 1.835 \\
\hline Boot Camp-Style & 2.528 & 540 & 0.012 & $6.34 \pm 2.69$ & $5.73 \pm 2.64$ & 0.134 & 1.070 \\
\hline Aquatic Exercise & 5.002 & 540 & $<0.001$ & $6.59 \pm 2.65$ & $5.39 \pm 2.69$ & 0.729 & 1.671 \\
\hline Mind-Body Movement & 5.548 & 540 & $<0.001$ & $6.61 \pm 2.58$ & $5.31 \pm 2.64$ & 0.842 & 1.764 \\
\hline Worker Incentive Programs & 5.098 & 540 & $<0.001$ & $6.56 \pm 2.62$ & $5.33 \pm 2.74$ & 0.757 & 1.706 \\
\hline $\begin{array}{l}\text { Boxing, Kickboxing \& Mixed } \\
\text { Martial Arts }\end{array}$ & 2.929 & 540 & 0.004 & $6.21 \pm 2.61$ & $5.52 \pm 2.62$ & 0.226 & 1.146 \\
\hline Dance-Based Work-Outs & 4.472 & 540 & $<0.001$ & $6.17 \pm 2.67$ & $5.13 \pm 2.54$ & 0.582 & 1.494 \\
\hline
\end{tabular}

$\mathrm{SD}$, standard deviation; $\mathrm{CI}$, confidence intervals. 
Table 4. Significant differences among the top 20 fitness trends and demographics

\begin{tabular}{|c|c|c|c|c|c|c|}
\hline \multirow{3}{*}{$\begin{array}{l}\text { Trend } \\
\text { Circuit Training }\end{array}$} & \multirow{2}{*}{\multicolumn{2}{|c|}{$\begin{array}{c}\text { Comparisons } \\
\text { Age }(y r s)\end{array}$}} & \multirow{3}{*}{$\begin{array}{c}\text { MD } \\
-1.013\end{array}$} & \multirow{3}{*}{$\begin{array}{c}\boldsymbol{p} \text { value } \\
0.024\end{array}$} & \multicolumn{2}{|c|}{$95 \% \mathrm{CI}$} \\
\hline & & & & & \multirow[b]{2}{*}{-1.96} & \multirow[b]{2}{*}{-0.07} \\
\hline & $18-21$ & $35-44$ & & & & \\
\hline & & $45-54$ & -1.361 & 0.003 & -2.43 & -0.30 \\
\hline \multirow[t]{5}{*}{ Strength Training (Free Weights) } & $18-21$ & $45-54$ & 1.153 & 0.026 & 0.07 & 2.23 \\
\hline & & $55-64$ & 2.083 & 0.011 & 0.27 & 3.89 \\
\hline & $22-34$ & $35-44$ & 0.593 & 0.047 & 0.00 & 1.18 \\
\hline & & $45-54$ & 1.029 & 0.002 & 0.25 & 1.80 \\
\hline & & $55-64$ & 1.960 & 0.007 & 0.31 & 3.61 \\
\hline \multirow[t]{3}{*}{ Outdoor Activities } & $18-21$ & $55-64$ & 2.819 & 0.006 & 0.47 & 5.17 \\
\hline & $22-34$ & $55-64$ & 2.574 & 0.006 & 0.44 & 4.71 \\
\hline & \multicolumn{2}{|c|}{ Education } & & & & \\
\hline \multirow[t]{2}{*}{ Children and Exercise } & Bachelor's & Doctorate & -0.965 & 0.016 & -1.83 & -0.10 \\
\hline & \multicolumn{2}{|c|}{ Experience (yrs) } & & & & \\
\hline Strength Training (Free Weights) & $0-5$ & $10-20$ & 0.748 & 0.020 & 0.08 & 1.42 \\
\hline \multirow[t]{2}{*}{ Fitness Programs for Older Adults } & $0-5$ & $20+$ & -0.882 & 0.011 & -1.63 & -0.14 \\
\hline & \multicolumn{2}{|c|}{ Work status } & & & & \\
\hline Exercise is Medicine & Full-time & Part-time & 0.548 & 0.003 & 0.16 & 0.94 \\
\hline Small Group Training & Full-time & Part-time & 0.500 & 0.010 & 0.09 & 0.91 \\
\hline Circuit Training & Full-time & Part-time & 0.687 & $<0.001$ & 0.26 & 1.12 \\
\hline \multirow[t]{2}{*}{ Strength Training (Free Weights) } & Full-time & Part-time & -0.637 & 0.002 & -1.08 & -0.19 \\
\hline & Full-time & Hourly basis & -0.952 & 0.027 & -1.82 & -0.08 \\
\hline \multirow[t]{2}{*}{ Outdoor Activities } & Full-time & Part-time & -0.775 & 0.003 & -1.35 & -0.21 \\
\hline & \multicolumn{2}{|c|}{ Career choice } & & & & \\
\hline Exercise for Weight Loss & First job & Second job & 0.535 & 0.025 & 0.05 & 1.02 \\
\hline Children and Exercise & First job & Third job & -0.954 & 0.032 & -1.85 & -0.06 \\
\hline Wearable Technology & First job & Second job & -0.764 & 0.004 & -1.34 & -0.19 \\
\hline Group Training & First job & Second job & -0.536 & 0.037 & -1.05 & -0.02 \\
\hline \multirow[t]{2}{*}{ Outdoor Activities } & First job & Second job & -0.997 & $<0.001$ & -1.60 & -0.39 \\
\hline & First job & Third job & -1.486 & 0.001 & -2.50 & -0.47 \\
\hline
\end{tabular}

$\mathrm{MD}$, mean difference; CI, confidence intervals. 
Table 5. Significant differences among the top 20 fitness trends and respondents' qualifications

\begin{tabular}{|c|c|c|c|c|c|c|c|}
\hline \multirow[b]{2}{*}{ Trend } & \multirow[b]{2}{*}{$t$} & \multirow[b]{2}{*}{ df } & \multirow[b]{2}{*}{$p$ value } & No & Yes & & \\
\hline & & & & \multicolumn{2}{|c|}{ Mean Score \pm SD } & \multicolumn{2}{|c|}{$95 \% \mathrm{CI}$} \\
\hline & \multicolumn{7}{|c|}{ Personal Training Qualification } \\
\hline Personal Training & -4.356 & 538 & $<0.001$ & $8.55 \pm 1.71$ & $9.12 \pm 1.34$ & -0.830 & -0.314 \\
\hline High-Intensity Interval Training & -5.404 & 535 & $<0.001$ & $7.96 \pm 1.96$ & $8.77 \pm 1.49$ & -1.094 & -0.511 \\
\hline Small Group Training & -2.151 & 540 & 0.032 & $8.13 \pm 1.91$ & $8.47 \pm 1.73$ & -0.651 & -0.029 \\
\hline Functional Fitness Training & -4.161 & 540 & $<0.001$ & $7.58 \pm 2.29$ & $7.06 \pm 2.80$ & -0.966 & -0.347 \\
\hline Circuit Training & -3.576 & 537 & $<0.001$ & $7.62 \pm 2.03$ & $8.21 \pm 1.84$ & -0.920 & -0.268 \\
\hline Fitness Programs for Older Adults & 2.480 & 456 & 0.013 & $7.81 \pm 2.00$ & $7.31 \pm 2.60$ & 0.104 & 0.901 \\
\hline Wearable Technology & 2.394 & 475 & 0.017 & $7.81 \pm 2.18$ & $7.30 \pm 2.66$ & 0.091 & 0.922 \\
\hline Outdoor Activities & 3.340 & 451 & 0.001 & $7.70 \pm 2.21$ & $6.95 \pm 2.92$ & 0.310 & 1.198 \\
\hline Post Rehabilitation Classes & 2.353 & 474 & 0.019 & $7.58 \pm 2.29$ & $7.06 \pm 2.80$ & 0.086 & 0.960 \\
\hline \multirow[t]{2}{*}{ Boutique Fitness Studios } & -3.533 & 540 & $<0.001$ & $6.96 \pm 2.42$ & $7.67 \pm 2.24$ & -1.109 & -0.316 \\
\hline & \multicolumn{7}{|c|}{ Exercise for Health Qualification } \\
\hline Exercise is Medicine & -2.997 & 314 & 0.003 & $8.68 \pm 1.85$ & $9.14 \pm 1.50$ & -0.770 & -0.160 \\
\hline Strength Training (Free Weights) & -2.134 & 540 & 0.033 & $7.51 \pm 2.02$ & $7.92 \pm 1.98$ & -0.798 & -0.033 \\
\hline Fitness Programs for Older Adults & -2.152 & 293 & 0.032 & $7.46 \pm 2.38$ & $7.91 \pm 2.06$ & -0.860 & -0.038 \\
\hline Children and Exercise & -2.583 & 540 & 0.010 & $7.29 \pm 2.27$ & $7.86 \pm 2.13$ & -0.987 & -0.134 \\
\hline Post Rehabilitation Classes & -3.254 & 299 & 0.001 & $7.14 \pm 2.63$ & $7.88 \pm 2.23$ & -1.186 & -0.292 \\
\hline \multirow[t]{2}{*}{ Clinical Integration/Medical Fitness } & -2.601 & 540 & 0.010 & $7.16 \pm 2.34$ & $7.74 \pm 2.17$ & $-1,017$ & -0.142 \\
\hline & \multicolumn{7}{|c|}{ Group Fitness Qualification } \\
\hline High-Intensity Interval Training & -3.608 & 305 & $<0.001$ & $8.20 \pm 1.91$ & $8.74 \pm 1.32$ & -0.837 & -0.246 \\
\hline Group Training & -2.903 & 540 & 0.004 & $7.43 \pm 2.20$ & $8.05 \pm 1.95$ & -1.056 & -0.203 \\
\hline Health/Wellness Coaching & -2.731 & 263 & 0.007 & $7.43 \pm 2.32$ & $7.98 \pm 1.84$ & -0.932 & -0.151 \\
\hline \multirow[t]{2}{*}{ Boutique Fitness Studios } & -2.544 & 252 & 0.012 & $7.15 \pm 2.45$ & $7.70 \pm 2.03$ & -0.973 & -0.124 \\
\hline & \multicolumn{7}{|c|}{ Youth Fitness Qualification } \\
\hline Personal Training & 2.275 & 63 & 0.026 & $8.88 \pm 1.50$ & $8.23 \pm 2.10$ & 0.079 & 1.221 \\
\hline Fitness Programs for Older Adults & -2.356 & 82 & 0.021 & $7.52 \pm 2.36$ & $8.12 \pm 1.76$ & -1.116 & -0.094 \\
\hline \multirow[t]{2}{*}{ Post Rehabilitation Classes } & -2.296 & 540 & 0.022 & $7.26 \pm 2.57$ & $8.07 \pm 2.15$ & -1.511 & -0.118 \\
\hline & \multicolumn{7}{|c|}{ Yoga Training Qualification } \\
\hline Personal Training & -2.732 & 62 & 0.008 & $8.77 \pm 1.61$ & $9.25 \pm 1.06$ & -0.829 & -0.129 \\
\hline \multirow[t]{2}{*}{ Post Rehabilitation Classes } & -2.862 & 540 & 0.004 & $7.25 \pm 2.53$ & $8.39 \pm 2.51$ & -1.918 & -0.357 \\
\hline & \multicolumn{7}{|c|}{ Aquatic Fitness Qualification } \\
\hline Outdoor Activities & -3.080 & 56 & 0.003 & $7.28 \pm 2.62$ & $8.24 \pm 1.86$ & -1.575 & -0.333 \\
\hline \multirow[t]{2}{*}{ Post Rehabilitation Classes } & -3.500 & 55 & 0.001 & $7.26 \pm 2.57$ & $8.36 \pm 1.90$ & -1.732 & -0.471 \\
\hline & \multicolumn{7}{|c|}{ Pre/postnatal Fitness Qualification } \\
\hline Exercise is Medicine & -2.572 & 48 & 0.013 & $8.77 \pm 1.80$ & $9.32 \pm 1.23$ & -0.994 & -0.122 \\
\hline Health/Wellness Coaching & -2.321 & 45 & 0.025 & $7.51 \pm 2.25$ & $8.22 \pm 1.73$ & -1.310 & -0.093 \\
\hline Outdoor Activities & -3.956 & 51 & $<0.001$ & $7.28 \pm 2.62$ & $8.43 \pm 1.62$ & -1.739 & -0.568 \\
\hline Post Rehabilitation Classes & -2.993 & 540 & 0.003 & $7.25 \pm 2.56$ & $8.54 \pm 1.98$ & -2.132 & -0.442 \\
\hline $\begin{array}{l}\text { Employing Certified Fitness } \\
\text { Professionals }\end{array}$ & -2.134 & 44 & 0.039 & $7.34 \pm 2.64$ & $8.16 \pm 2.24$ & -1.605 & -0.046 \\
\hline \multirow[t]{2}{*}{ Clinical Integration/Medical Fitness } & -2.702 & 540 & 0.007 & $7.24 \pm 2.30$ & $8.30 \pm 2.25$ & -1.823 & -0.288 \\
\hline & \multicolumn{7}{|c|}{ Active Aging Fitness Qualification } \\
\hline Children and Exercise & -2.381 & 540 & 0.018 & $7.39 \pm 2.26$ & $8.44 \pm 1.81$ & -1.920 & -0.184 \\
\hline Post Rehabilitation Classes & -2.753 & 31 & 0.010 & $7.29 \pm 2.56$ & $8.33 \pm 1.88$ & -1.817 & -0.271 \\
\hline
\end{tabular}

$\mathrm{SD}$, standard deviation; $\mathrm{CI}$, confidence intervals. 


\section{Discussion}

The Greek survey of fitness trends was conducted for the first time aiming to support all involved stakeholders and to recognize the current status of the industry regarding the most popular exercise modes and programs. Moreover, it may assist practitioners, entrepreneurs, and educators to enhance customer engagement and experience through applicable strategies in the health and fitness industry. In Greece, personal training, exercise is medicine, high-intensity interval training (HIIT), small group training, and functional fitness were identified as the top 5 trends for 2021, respectively. Interestingly, 35\% of top 20 selections are health-oriented fitness trends associated with exercise for health and special populations, including seniors, children and adults with chronic conditions. All technology-oriented fitness trends (e.g., outcome measurements, online training, mobile exercise apps, virtual training, and electrical muscle stimulation training) except wearable technology are not yet very attractive. Likewise, mind/body fitness-related trends such as Pilates, Yoga, and Tai Chi were not included in the top 20 demonstrating low popularity in Greece.

The present findings also show that female survey respondents reported higher scores than male for almost $70 \%$ (28/41) of fitness trends and only low-cost and budget gyms received a higher score by male respondents (Table 3). This outcome cannot be explained here, but it may indicate that gender may be a critical demographic characteristic among health and fitness industry stakeholders while underlining the need for further investigation in the future. In terms of the effect of various demographics on the fitness trends popularity, we observed that circuit training was more attractive among middle-aged respondents (35-54 years) compared to young participants (18-21 years). On the other side, strength training with free weights seems to be more popular to younger (18-34 years) than older respondents (35-64 years). Additionally, participants aged from 18 to 34 years reported a remarkably higher mean score for outdoor activities than older participants (55-64 years). Interestingly, education as a demographic characteristic showed meaningful differences only for children and exercise that was ranked higher among doctoral degree holders than those holding a bachelor's degree. Moreover, strength training with free weights appears to be more important to survey respondents with less professional experience ( $0-5$ years vs. 10-20 years) while fitness programs for older adults were more popular among very experienced industry stakeholders $(20+$ years vs. 0-5 years). Examining the role of work status in the attractiveness of fitness trends nationwide, we observed that full-time demonstrates impactful differences compared to part-time for exercise is medicine, small group training, and circuit training. On the other side, part-time showed higher applicability for strength training with free weights and outdoor activities among respondents. Noticeably, group training, children and exercise, wearable technology, and outdoor activities were ranked significantly higher by respondents who stated their occupation in the health and fitness sector as the second or third career choice. In contrast, only exercise for weight loss was selected as an important fitness trend by survey participants who stated their involvement in the industry as the first career choice. Furthermore, our main findings indicate that all those trends presented meaningful differences among various qualifications were ranked higher by holders of qualifications in exercise for health, group fitness, active aging fitness, yoga training, aquatic fitness, and pre/postnatal fitness. (Table 5). Such a result was not observed in participants holding a qualification in personal training. More specifically, five out of top 20 trends (e.g., personal training, high-intensity interval training, small group training, circuit training, and boutique fitness studios) were ranked higher, but functional fitness, fitness programs for older adults, outdoor activities, and post rehabilitation classes were ranked lower compared to the mean score given by unqualified survey participants.

The main results of the first-ever Greek survey on fitness trends using the ACSM's methodology [14] show many comparable points with other recently published studies investigating fitness trends in various regions [15]. Specifically, personal training was ranked \#1 in the present study, which is an outcome totally aligned with the primary finding observed in Europe [16]. In addition, this particular trend appears to be popular worldwide as the fifth most attractive fitness trend [14] while it was ranked \#3 in North America [15], \#4 in Brazil and Argentina [18], \#7 in Spain [19], and \#8 in China [17]. Fitness services focused on personal training seem to be significantly popular for Europeans and thus almost 3 out of 4 of the offered exercise services are traditional one-on-one training sessions [20]. Furthermore, the occupation of personal trainer has been reported as the second most promising working role among European exercise professionals [21]. These findings may confirm that the growth of the health and fitness industry is linked to those services that are capable of improving the customers exercise experience through professionalism, individualization, and innovation [3]. As such, personal training space seems to be very attractive for all involved fitness industry stakeholders aiming to increase service quality, exercise motivation as well as members' satisfaction and loyalty [3].

HIIT (\#3), small group training (\#4), functional fitness training (\#5), body weight training (\#6), and circuit training (\#7) are trends that seem to be attractive not only in Greece but across the world [14]. Their popularity may be systematically increasing in the global fitness industry because these particular trends are considered the principal services in boutique fitness studios (\#19), which 
has been documented as an emerging fitness setting [22]. Moreover, such studios mainly offering targeted services are considered a promising workspace for fitness practitioners in Europe [21]. These facts support the evidence that fitness industry is changing by shaping a different landscape highly impacted by innovation, market challenges and local socioeconomic status within a very competitive environment [3]. Notably, business models have changed over the years in the Greek health and fitness industry mainly because of the attempt to adapt to this changing landscape due to a long-lasting socioeconomic crisis since 2009 [23]. Thus, emerging market segments such as personal training and boutique fitness studios as well as low-cost and budget gyms (box gyms) appear to be some new perspectives on customer engagement and experience [22,24]. According to our findings, box gyms (\#31) are not yet considered as a very popular fitness setting among Greek practitioners and industry stakeholders. However, it has been observed a direct and positive relationship between perceived quality and service convenience on perceived value, satisfaction and loyalty in members of low-cost fitness centers in Spain [25]. A plethora of studies show that HIIT may be a safe, effective and time-efficient exercise approach for improving cardiometabolic health and physical performance in general, clinical and athletic populations [26]. In contrast, there is evidence that it is not clear if HIIT can be a feasible and enjoyable exercise approach for the masses as an additional public health policy supporting the benefits of long-term exercise using such an aggressive training modality [27,28]. Additionally, recent research shows that circuit-type exercise programs incorporating HIIT, functional fitness and bodyweight training into a small-group gym getting elicit favorable changes in several physiological $[29,30]$ and psychological [31] indicators of previously inactive, middle-aged women with overweight or obesity. On the other side, large multipurpose facilities are still popular in Greece and may be this fact supports the finding that traditional exercise modes such as strength training with free weights (\#9) and group training (\#12) are considered attractive at both national and international level [15]. Specifically, group training classes delivering HIIT, functional fitness, and bodyweight programs are considered some of the principal services of the multipurpose fitness centers in Europe [32]. Furthermore, resistance training has been extensively documented as a fundamental component of every structured physical exercise program for apparently healthy adults of all ages [33].

In the present study, seven out of top 20 selections are health-oriented trends showing that exercise for health focusing on targeted populations may be an area with great potential for the future. Specifically, exercise is medicine (\#2), exercise for weight loss (\#7), fitness programs for older adults (\#10), health/wellness coaching
(\#13), children and exercise (\#14), post rehabilitation classes (\#17), and clinical integration/medical fitness (\#18) seem to be attractive fields for practitioners, educators, and entrepreneurs in the Greek fitness sector. Considering the fact that several lifestyle-related chronic diseases are currently increasing in Greece [9], our findings these outcomes may be an interesting sign for the future state of the national fitness industry highlighting the pivotal role of fitness practitioners and businesses in public health. Additionally, these outcomes corroborate the majority of the literature showing that $25 \%-35 \%$ of top 20 fitness trends are associated with exercise training for special populations and thus health-related trends systematically gain popularity in the global fitness industry [14,16-18]. Taking these findings into account, it seems that the local fitness industry supports the scientific evidence underlining the critical role of structured exercise in health, performance and quality of life of individuals suffered from chronic conditions [34], including adults [35], and children with obesity [36] as well as elderly [37] and persons who regularly exercise in a clinical setting [13]. Specifically, our results may indicate that obesity and aging as two sides of the same coin promoting numerous comorbidities and age-related chronic conditions [38] appear to be attractive areas for the Greek fitness industry stakeholders. Additionally, exercise training for children as a trend ranked in the top 20 in the present study, it appears to be a key area for business and professional development in the local fitness sector supporting the necessity of regular physical activity in youth due to exercise deficit disorder [39] and obesity [8] observed at an alarming rate in children and adolescents.

Interestingly, technology-oriented fitness trends such as outcome measurements (\#27), online training (\#30), mobile exercise apps (\#34), virtual training (\#40), and electrical muscle stimulation training (\#41) were not in the top 20. Only wearable technology (\#11) demonstrates a noteworthy popularity that is aligned with data recently reported from similar surveys conducted in North America [14] and China [17], but not in Europe [16] and South America [18]. Despite the fact the digitalization is currently changing the fitness sector by transforming the old-school business models widely used in the gyms and studios [24], this observation may highlight the role of socioeconomic status in the magnitude of use of technology. In other words, digital fitness services and programs may be affected by the lack of financial resources due to the current economic crisis in Greece [40]. Furthermore, considering that individualized exercise training clearly shows high attractiveness among respondents in Greece, it is notable that mind/body-oriented fitness trends such as Pilates (\#22), Yoga (\#29), and Tai Chi (\#36) demonstrates low popularity resulting into their absence from the top 20 . Such alternative exercise modes also are not very popular in other regions [15] and only Yoga was ranked in the top 
20 in North America (\#14) [14], China (\#15) [17], and Europe (\#20) [16]. Moreover, even though group training (\#12) appears to be an attractive exercise mode in the Greek health and fitness industry, specialized group training programs such as dance-(\#39), boxing- (\#38), and aquatic-based (\#35) workouts show limited applicability and thus they were ranked outside the top 20. Likewise, these particular trends present low attractiveness among fitness practitioners worldwide [15].

Observing the increased popularity of personal training and health-related fitness trends in the Greek survey, it is an outcome that may support the evidence that a substantial large percentage of exercisers nationwide are individuals with health issues, poor functional capacity and potential physical limitations [9] that may restrict their participation in such exercise modes. Hence, an inclusive environment for the masses may be needed in order to engage populations with no exercise experience in regular movement and adapted fitness programs. Additionally, fitness trends related to professional, ethical and legal issues such as employing certified fitness professionals (\#15) and licensure for fitness professionals (\#20) seem to gain an increasing popularity lately not only in Greece but also in Spain [19], North America [14], Europe [16] and South America [18], but not too much in Asia [17]. The rationale for well-equipped exercise professionals is systematically growing because of the increased prevalence of populations struggling with numerous chronic conditions that reduce quality of life and increase mortality [5,8]. As such, the changing landscape of the fitness industry may be linked to the current epidemiological statistics underlining the necessity of qualified practitioners capable of supporting underserved markets such as older adults, individuals with controlled diseases, children/adolescents with obesity and persons that primarily need personalized services [41]. The qualification of personal trainer has been reported as one of the most important professional credentials for the European fitness practitioners [16,21] and personal training services are and will continue to be in increasing demand in Europe [20]. These facts are aligned with our findings showing that personal training is \#1 trend in Greece for 2021. Furthermore, a review of the literature shows that the role of fitness professionals in public health [42] is currently changing and more specifically, exercise physiologists may play a critical role in the management of noncommunicable chronic diseases as essential members of interdisciplinary teams [43]. These facts may support the collaboration between qualified exercise professionals and physicians in order to promote the mission of the global initiative of Exercise is Medicine ${ }^{\circledR}$ established by ACSM aiming to enhance the systematically growing rationale of exercise prescription by physicians [44]. Lastly, the first-ever Greek survey of fitness trends may highlight the demand for regulation and licensing requirements for exercise professionals as widely used by other professions both nationwide and around the world.

\subsection{Strengths and Limitations}

To our best of knowledge, this is the first study examining potential differences between fitness trends and demographics of various stakeholders in the health and fitness sector. The major limitation of the present study is the language barrier playing a critical role in the ease of use, usability and respondents experiences while using the online questionnaire that may affect the sample size. However, 542 responses are considered a large sample size for a national survey conducted in Greece. It is notable that significantly larger fitness markets such as the North America, Europe, China, South America, and Spain collected data from 1,995 [14], 1,001 [16], 1,827 [17], 894 [18], and 513 [19] respondents, respectively. Considering that our survey conducted during the coronavirus pandemic, we could conclude that our findings may be affected by these challenging and uncertain circumstances. Hence, a similar survey should run next year aiming to present comparative data on fitness trends in Greece for 2021 and 2022. Furthermore, even though English is considered a popular foreign language among the health and fitness industry stakeholders in Greece, it may be challenging for locals to complete a detailed questionnaire exclusively provided in English. Although the return rate is considered notably high $(15.5 \%)$ compared to other international studies on fitness trends [14,17-19], a Greek survey may be suggested for future research aiming to investigate the same topic by recruiting more respondents. Furthermore, no incentive (financial or material) was offered to respondents in the Greek study as similarly applied in other international surveys [16-19] except the ACSM's worldwide survey [14], which may be an additional tool for increasing the number of responses in surveys of this kind. Finally, additional reminders (only one reminder was used) may increase both the return rate and sample size in a future study.

\section{Conclusions}

In summary, personal training was ranked in the \#1 spot for 2021, which is a result absolutely aligned with previous findings from a European study on fitness trends [16]. This particular trend has been systematically reported as one of the most attractive and popular options among exercisers and fitness professionals at both European [20] and worldwide [14] level. The present investigation of the Greek fitness trends provides the possibility to examine potential comparisons among Greece and other regions aiming to provide data regarding the popularity and attractiveness of specific fitness services, programs and credentialing at regional and 
global level. Considering that sedentary behavior is systematically increasing in the past two decades throughout Europe [45], this survey as the first study of its kind aims to guide and support all involved national industry stakeholders on how to develop and apply safe, efficient, and attractive physical activity and exercise concepts relevant to current top fitness trends. The interpretation of these results into valid solutions that are targeted to promote a positive exercise experience for consumers will help the local fitness sector continue its principal work by improving its services through innovation and evidence-based practices while fighting against the inactivity epidemic. Lastly, further research is needed in this area examining national fitness trends using the ACSM's methodology in more countries because to the best of our knowledge, only Spain [19] and Ukraine [46] present relevant data to date. Such research attempts may support the vision of the health and fitness sector for further growth and innovation promoting fitness as mainstream physical activity or sport around the globe [47].

\section{Acknowledgements}

The authors would like to express their gratitude to all participants for their contribution and more specifically to Olympia Zarampouka for her support and help with the participant recruitment procedure.

\section{REFERENCES}

[1] Deloitte. European Health \& Fitness Market Report 2020. EuropeActive, Brussels, Belgium, 2020.

[2] International Health, Racquet and SportsClub Association. The 2019 IHRSA Global Report. IHRSA Publications, Boston, MA, USA, 2019.

[3] Middelkamp J, Rutgers H. Growing the Fitness Sector Through Innovation. EuropeActive, Brussels, Belgium \& BlackBoxPublishers, Den Bosch, the Netherlands, 2016.

[4] Guthold R, Stevens GA, Riley LM, Bull FC. Worldwide trends in insufficient physical activity from 2001 to 2016: a pooled analysis of 358 population-based surveys with 1.9 million participants. Lancet Glob Health 6(10): e1077e1086, 2018.

[5] NCD Risk Factor Collaboration (NCD-RisC). Worldwide trends in body-mass index, underweight, overweight, and obesity from 1975 to 2016: a pooled analysis of 2416 population-based measurement studies in 128.9 million children, adolescents, and adults. Lancet 390(10113): 2627 2642, 2017.

[6] International Health, Racquet and SportsClub Association. The 2013 IHRSA European Health Club Report: Size and Scope of the Fitness Industry. IHRSA Publications, Boston, MA, USA, 2013.
[7] International Health, Racquet and SportsClub Association. The 2008 IHRSA European Market Report: The size and scope of the health club industry. IHRSA Publications, Boston, MA, USA, 2008.

[8] World Health Organization. Noncommunicable diseases country profiles 2018, Geneva, Switzerland. Online available from https://apps.who.int/iris/handle/10665/27451 2. [Accessed: 24 September 2018].

[9] World Health Organization. Greece: Profile of Health \& Well-being. WHO Regional Office for Europe, Copenhagen, Denmark. Online available from https://www.euro.who.int/ en/publications/abstracts/greece.-profile-of-health-and-well -being-2016 [Accessed: 12 March 2016].

[10] European Commision. Special Eurobarometer 472 Report: Sport and Physical Activity. European Commission, Brussels, Belgium, 2018.

[11] World Health Organization. Physical Activity Factsheets for the 29 European Union Member States of the WHO European Region. WHO Regional Office for Europe, Copenhagen, Denmark. Online available from https://www.euro.who.int/en/health-topics/disease-preventi on/physical-activity/publications/2018/factsheets-on-healthenhancing-physical-activity-in-the-28-eu-member-states-ofthe-who-european-region. [Accessed: 22 September 2018].

[12] American College of Sports Medicine. ACSM's Guidelines for Exercise Testing and Prescription 10th ed. Lippincott Williams \& Wilkins, Philadelphia, PA, USA, 2017.

[13] Muth ND, Vargo K, Bryant CX. The Role of the Fitness Professional in the Clinical Setting. Curr Sports Med Rep 14(4): 301-312, 2015.

[14] Thompson WR. Worldwide survey of fitness trends for 2020. ACSMs Health Fit J 23(6): 10-18, 2019.

[15] Kercher VM, Feito Y, Yates B. Regional Comparisons: The Worldwide Survey of Fitness Trends. ACSMs Health Fit J 23(6): 41-48, 2019.

[16] Batrakoulis A. European survey of fitness trends for 2020. ACSMs Health Fit J 23(6): 28-35, 2019.

[17] Li Y-M, Han J, Liu Y, Wang R, Wang R, Wu X-P, Cao Z-B. China survey of fitness trends for 2020. ACSMs Health Fit J 23(6): 19-27, 2019.

[18] Costa Amaral P, Palma D. Brazil and Argentina survey of fitness trends for 2020. ACSMs Health Fit J 23(6): 36-40, 2019.

[19] Cámara M, Valcarce M, Veiga OL. National survey of fitness trends in Spain for 2020. Retos 37: 427-433, 2020.

[20] Gronau N, Titze G. Personal Training in Europe. EuropeActive, Brussels, Belgium \& BlackBoxPublishers, Den Bosch, the Netherlands, 2018.

[21] Batrakoulis A, Rieger T, Santos Rocha R. The relationship between special populations and credentials for the European fitness professionals. 28th Symposium of the International Council for Physical Activity and Fitness Research (ICPAFR), Lithuanian Sports University, August 24-27, Kaunas, Lithuania, p. 27, 2016.

[22] International Health, Racquet and SportsClub Association. The 2019 IHRSA Health Club Consumer Report. IHRSA 
Publications, Boston, MA, USA, 2019.

[23] Stylianidis S, Souliotis K. The impact of the long-lasting socioeconomic crisis in Greece. BJPsych Int 16(1): 16-18, 2019.

[24] Middelkamp J, Rutgers H. Customer engagement and experience in the fitness sector. EuropeActive, Brussels, Belgium \& BlackBoxPublishers, Den Bosch, the Netherlands, 2017.

[25] García-Fernándeza J, Gálvez-Ruíz P, Fernández-Gavira J, Vélez-Colón L, Pitts B, Bernal-Garcíae A. The effects of service convenience and perceived quality on perceived value, satisfaction and loyalty in low-cost fitness centers. Sport Manage Rev 21(3): 250-262, 2018.

[26] Kilpatrick MW, Jung ME, Little JP. High-intensity interval training: A review of physiological and psychological responses. ACSMs Health Fit J 18(5): 11-16, 2014.

[27] Biddle SJH, Batterham AM. High-intensity interval exercise training for public health: a big HIT or shall we HIT it on the head? Int J Behav Nutr Phys Act 12: 95, 2015.

[28] Gray SR, Ferguson C, Birch K, Forrest LJ, Gill JMR. High-intensity interval training: key data needed to bridge the gap from laboratory to public health policy. $\mathrm{Br} J$ Sports Med 50(20): 1231-1232, 2016.

[29] Batrakoulis A. Jamurtas AZ, Georgakouli K, Draganidis D, Deli CK, Papanikolaou K, Avloniti A, Chatzinikolaou A, Leontsini D, Tsimeas P, Comoutos N, Bouglas V, Michalopoulou M, Fatouros IG. High intensity, circuit-type integrated neuromuscular training alters energy balance and reduces body mass and fat in obese women: A 10-month training-detraining randomized controlled trial. PLOS ONE 13(8): e0202390, 2018.

[30] Batrakoulis A, Tsimeas P, Deli CK, Vlachopoulos D, Ubago-Guisado E, Poulios A, Chatzinikolaou A, Draganidis D, Papanikolaou P, Georgakouli P. Batsilas D, Gracia-Marco L, Jamurtas AZ, Fatouros IG. (2020). Hybrid Neuromuscular Training Promotes Musculoskeletal Adaptations in Inactive Overweight and Obese Women: A Training-Detraining

Randomized Controlled Trial. Journal of Sports Sciences 2020, doi: 10.1080/02640414.2020.1830 543. [Epub ahead of print].

[31] Batrakoulis A, Loules G, Georgakouli K, Tsimeas P, Draganidis D, Chatzinikolaou A, Papanikolaou K, Deli CK, Syrou N, Comoutos N, Theodorakis Y, Jamurtas AZ, Fatouros IG. High-intensity interval neuromuscular training promotes exercise behavioral regulation, adherence and weight loss in inactive obese women. Eur J Sport Sci 20(6): 783-792, 2020.

[32] Middelkamp J, Wolfhagen P, Eemstra J. Group Fitness in Europe. BlackBoxPublishers, Den Bosch, the Netherlands, 2020.

[33] Westcott WL. Build muscle, improve health: Benefits associated with resistance exercise. ACSMs Health Fit $J$ 19(4): 22-27, 2015.

[34] Thompson WR, Sallis R, Joy E, Jaworski CA, Stuhr RM, Trilk JL. Exercise is Medicine. Am J Lifestyle Med 2020, doi: 10.1177/1559827620912192 [Epub ahead of print].

[35] Garber CE. The health benefits of exercise in overweight and obese patients. Curr Sports Med Rep 18(8): 287-291, 2019.

[36] Faigenbaum AD, MacDonald JP, Carvalho C, Rebullido TR. The pediatric inactivity triad: A triple jeopardy for modern day youth. ACSMs Health Fit J 24(4): 10-17, 2020.

[37] Dipietro L, Campbell WW, Buchner DM, Erickson KI, Powell KE, Bloodgood B, Hughes T, Day KR, Piercy KL, Vaux-Bjerke A, Olson RD, 2018 Physical Activity Guidelines Committee. Physical activity, injurious falls, and physical function in aging: An umbrella review. Med Sci Sports Exerc 51(6): 1303-1313, 2019.

[38] Tam BT, Morais JA, Santosa S. Obesity and ageing: Two sides of the same coin. Obes Rev 21(4): e12991, 2020.

[39] Walker G, Stracciolini A, Faigenbaum A, Myer G. Physical inactivity in youth: Can exercise deficit disorder alter the way we view preventative care? ACSMs Health Fit J 22(2): 42-46, 2018

[40] Zavras D, Tsiantou V, Pavi E, Mylona K, Kyriopoulos J. Impact of economic crisis and other demographic and socio-economic factors on self-rated health in Greece. Eur J Public Health 23(2): 206-210, 2013.

[41] Batrakoulis A. The interaction between metabolic disorders and professional credentials. Personal Trainer Quarterly 3(1): 18-20, 2016.

[42] De Lyon ATC, Neville RD, Armour KM. The role of fitness professionals in public health: A review of the literature. Quest 69(3): 313-330, 2017.

[43] Soan EJ, Street SJ, Brownie SM, Hills AP. Exercise physiologists: essential players in interdisciplinary teams for noncommunicable chronic disease management. $J$ Multidiscip Healthc 7: 65-68, 2014.

[44] Sallis R. Exercise is medicine: a call to action for physicians to assess and prescribe exercise. Phys Sportmed 43(1): 2226,2015 .

[45] López-Valenciano A, Mayo X, Liguori G, Copeland RJ, Lamb M, Jimenez A. Changes in sedentary behaviour in European Union adults between 2002 and 2017. BMC Public Health 20: 1206, 2020.

[46] Vorobiova A. World and national fitness trends 2019. Sport Science and Human Health 1(1): 62-69, 2019.

[47] Middelkamp J, Rutgers H. HORIZON 2030 - The future of the health and fitness sector. EuropeActive, Brussels, Belgium \& BlackBoxPublishers, Den Bosch, the Netherlands, 2020. 